\title{
MammoSite brachytherapy: evolution and current status
}

\author{
R. Rabinovitch, A. Schwer \\ Anschutz Centers for Advanced Medicine-Fitzsimons, University of Colorado Hospital, Aurora, CO, USA.
}

\begin{abstract}
Brachytherapy treatment consists of the insertion of radioactive sources into tissue to deliver radiotherapy directly to the tumor. Early treatment utilizing this method required intra-operative placement of 15-20 catheters by the radiation oncologist, a highly specialized procedure which limited its widespread use despite encouraging results from the first clinical trial and the benefits to the patient of receiving radiation treatment to only $2 \mathrm{~cm}$ of tissue beyond the borders of the lumpectomy cavity and the ability to complete the radiotherapy component of breast conserving treatment in 4-5 days, instead of the usual 4-6 weeks. The MammoSite $\mathbb{R}$ Radiation Therapy System allows either a radiation oncologist or a surgeon to implant a much simplified brachytherapy device, thus increasing the number of patients who can receive this treatment. The device, which received FDA approval in May 2002, works by creating a cavity inside the lumpectomy site via a balloon. A high dose-rate brachytherapy source is inserted into this cavity, evenly irradiating the tissue at highest risk of containing residual cancer cells. Data collected since its approval show that MammoSite offers tumor control equal to traditional radiotherapy with fewer side effects of radiation exposure to nearby tissues. However, the invasive nature and high cost of this treatment mean that MammoSite should not be seen as the sole future direction of radiotherapy treatment delivery methods.
\end{abstract}

Keywords: Brachytherapy; MammoSite; Radiation therapy

The MammoSite ${ }^{\circledR}$ Radiation Therapy System (RTS) is a novel brachytherapy applicator initially designed and manufactured by Proxima Therapeutics (now Cytec Surgical Products) to simplify and hence enhance the availability of breast brachytherapy to women with early stage breast cancer.

\section{Background of breast brachytherapy}

In the early 1990s, interest in utilizing brachytherapy (the insertion of radioactive sources into tissue to deliver radiotherapy directly to the tumor) through a multi-catheter technique became an exciting new

Correspondence to: R. Rabinovitch, MD, Anschutz Centers for Advanced Medicine-Fitzsimons, University of Colorado Hospital, Aurora, CO 80045 USA. E-mail: rachel.rabinovitch@uchsc.edu

Received: 09/01/06

Accepted: 19/04/05

First published online 05/10/06

BCO/365/2005/FO approach in the treatment of early stage breast cancers. Unlike standard conventional breast radiotherapy, this approach treats only $2 \mathrm{~cm}$ of tissue beyond the borders of the lumpectomy cavity instead of the entire breast. The advantage of brachytherapy was the ability to complete the radiotherapy component of breast conserving treatment in 4-5 days, instead of the usual $4-6$ weeks. The first brachytherapy technique developed required intra-operative placement of 15-20 catheters by the radiation oncologist in a highly specialized procedure. Early single institution results, pioneered in the United States by Dr. Robert Kuske, then from the Oschner Clinic, were promising. Based on this initial data, the Radiation Therapy Oncology Group (RTOG) conducted a phase II trial between 1997 and 2000 evaluating the feasibility and efficacy of partial breast brachytherapy utilizing the multi-catheter technique in 100 patients (RTOG 95-17).

Although the trial was successfully completed, it was observed that relatively few institutions 
participated in this trial. This was attributed to the assumption that most radiation oncologists were not comfortable with this complex form of brachytherapy and not willing to commit to the extensive procedure time; physics support for this multi-catheter technique was also quite sophisticated and not consistently available at all practice sites.

\section{Development of the MammoSite RTS}

Proxima Therapeutics (acquired in 2005 by Cytec Surgical Products) appreciated the potential importance and popularity of an effective 5-day brachytherapy treatment for early stage breast cancer and noted the barriers of the complex multicatheter approach. The company therefore developed a novel and elegantly simple brachytherapy device which could be easily implanted by either a radiation oncologist or a surgeon.

The device consists of a double lumen catheter embedded in an inflatable spherical balloon at one end (Fig. 1). Following insertion of the balloon into the lumpectomy cavity, the balloon is inflated via one of the lumens with sterile water and contrast material. The expansion of the balloon fills the lumpectomy cavity and symmetrically expands the cavity and compresses surrounding breast tissue. After treatment planning, a high dose-rate brachytherapy source is inserted through the second lumen, evenly irradiating the tissue at highest risk of containing residual cancer cells - the tissue surrounding the symmetrically expanded balloon. The prescription dose is delivered $1 \mathrm{~cm}$ beyond the surface of the balloon.

The initial MammoSite design utilized a spherical balloon, with a single dwell position of radioactivity. (The company has since developed balloons of several shapes and sizes, with the ability to alter source loading options and accommodate variations in lumpectomy cavity size and shape.) This greatly minimizes the physics planning and sophistication required for successful treatment delivery. Treatment is delivered twice daily for 5 consecutive days, and utilizes the same dose schedule designed for the multi-catheter technique - 340 cGy/fraction, prescribed to $1 \mathrm{~cm}$ beyond the surface of the balloon. Upon completion of the 10th treatment, the balloon is deflated and simply extracted from the patient.

After testing the device in 43 patients between May 2000 and October 2001 to determine the product's safety and performance, the device received FDA approval in May 2002 and became commercially available. The FDA requires the product insert to contain the following black box warning: 'The safety and effectiveness of the MammoSite ${ }^{\circledR}$ RTS as a replacement for whole breast irradiation in the treatment of breast cancer has not been established.'

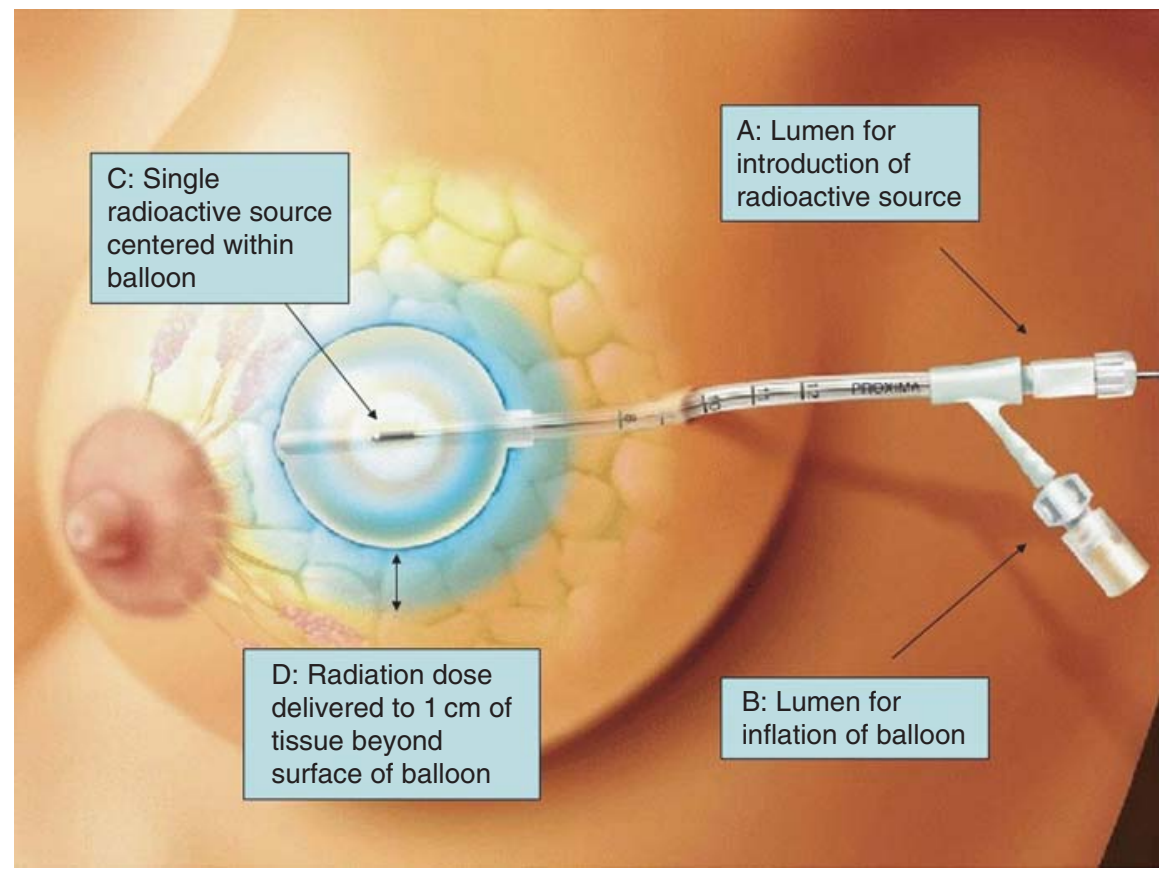

Figure 1.

Graphic display of the MammoSite RTS. Note the double lumen catheter (A and B), evenly expanded spherical balloon, and the single radiation source positioned in the center of the balloon (C). The prescribed radiation dose is delivered to the $1 \mathrm{~cm}$ of compressed breast tissue surrounding the balloon (D). (Image courtesy of Cytec Corporation). 


\section{MammoSite: post-FDA approval}

Over 12000 women have been treated with MammoSite brachytherapy since the device's FDA approval, attesting to the enthusiasm embraced by both patient and physician of a 5-day treatment approach for radiotherapy in early stage breast cancer. The company in coordination with the American Society of Breast Surgeons (ASBS) [1] has entered 1449 patients treated around the country in a registry trial designed to assess and record the toxicity, cosmesis, and tumor control rates of MammoSite brachytherapy in a larger population of patients [2].

\section{Patient selection criteria}

The American Brachytherapy Society and the ASBS have each developed consensus recommendations regarding optimal patient selection criteria for partial breast irradiation (PBI) (see Table 1). NSABP B-39/ RTOG 0413 [3] is a national cooperative group trial which is comparing conventional whole breast irradiation to any one of 3 forms of PBI (multi-catheter brachytherapy, MammoSite brachytherapy, or external beam 3D conformal radiotherapy); this trial is currently open and accruing patients, with the goal of enrolling 3000 women with early stage breast cancer over 3 years. This protocol allows patients with unifocal breast cancer (DCIS or invasive disease) up to $3 \mathrm{~cm}$ in size and with up to 3 positive lymph nodes to participate.

\section{Technical considerations}

The MammoSite RTS is inserted into the lumpectomy cavity either during the same procedure (open cavity technique) or days later (closed cavity technique). After insertion, the balloon is maximally inflated with sterile water and contrast material (to allow for visualization of the balloon on X-ray imaging). The device and surrounding breast tissues then undergo CT evaluation for determination of the presence of surrounding air or fluid between the balloon and targeted tissue, either of which can render the dosimetry suboptimal. Excessive air is often resorbed by the body over the
24-48 hours after placement, and re-evaluation is indicated at that time. Balloon symmetry is necessary to ensure appropriate dosimetry; a minimum of $7 \mathrm{~mm}$ of tissue between the balloon and skin surfaces is required to prevent excessive skin dosing.

\section{Limitations of the MammoSite RTS}

Placement of the MammoSite RTS is an invasive device requiring sterile technique and local anesthetic at a minimum. The device remains within the patient for typically 8-10 days. Pain and infection are risks associated with an invasive treatment approach not relevant for conventional whole breast radiotherapy or other external beam radiotherapy techniques. Once placed, the device may be rendered unsuitable for treatment due to a variety of factors which primarily include: excessive surrounding fluid or air, balloon asymmetry, and inadequate tissue thickness between balloon surface and skin. The rates of these events, requiring the device to be removed and other radiotherapy treatment options to be considered, are largely unreported. Despite these potential obstacles, some postulate that the intense dose gradients associated with brachytherapy treatment may play an important role in achieving good tumor control, which would not be replicated with the uniform dosimetry associated with external beam techniques.

The recent development of external beam PBI treatment techniques (either 3D or Intensity Modulated Radiotherapy) have the advantages of being both non-invasive, and able to avoid all of the technical barriers encountered with the MammoSite RTS. However, there is even less published data on the toxicity and efficacy of external beam PBI when compared to MammoSite, as it is the most recently developed approach.

\section{Toxicity}

The toxicity of MammoSite brachytherapy is of paramount interest. Infection rates vary from $3.7 \%$ to $16 \%$, with the largest series from the ASBS registry trial reporting a middle figure of $8 \%[5,6,8]$. Other toxicities include erythema (25-75\%), desquamation (7-13\%),

Table 1. Patient selection recommendations.

\begin{tabular}{llll}
\hline & ABS recommendation (1) & ASBS recommendation (2) & NSABP B-39/RTOG 0413 eligibility (3) \\
\hline Age & $>45$ & $>50$ & $\geqslant 18$ \\
Tstage & Unifocal invasive ductal carcinoma & Invasive Ductal Carcinoma or & $\begin{array}{l}\text { Invasive Ductal or Lobular Carcinoma } \\
\text { or DCIS } \\
\end{array}$ \\
Tumor size & $\leqslant 3 \mathrm{~cm}$ & DCIS & $\leqslant 3 \mathrm{~cm}$ \\
Surgical margins & No tumor at margin & $\leqslant 2 \mathrm{~cm}$ & No tumor at margin \\
Nodal status & N0 & $\geqslant 2 \mathrm{~mm}$ margins & N0-N1 \\
\hline
\end{tabular}


fat necrosis $(7 \%)$, breast edema (3-15\%), fibrosis (6-11\%), and telangectasias (10\%). Contributing to the difficulty in interpreting the available toxicity data is the lack of uniformity in toxicity definitions.

\section{Costs}

Despite the shortened treatment time and lesser number of treatments with MammoSite brachytherapy compared to whole breast radiotherapy, MammoSite brachytherapy is actually more expensive. This is largely due to the costs associated with high doserate brachytherapy delivery. For an extensive cost comparison analysis of numerous breast radiation techniques [4].

\section{Cosmesis}

Cosmetic outcome of the treated breast is highly subjective. Nevertheless, it is an important endpoint in breast conserving therapy, particularly with a new treatment approach. Numerous authors have reported on the rates of 'good/excellent cosmesis' in their MammoSite-treated cohorts, and results range from $80 \%$ to $95 \%$ [5-8]. These good-excellent results are not based on randomized comparisons to conventional whole breast radiotherapy, but are sufficiently high to maintain confidence in the MammoSite application. The results of NSABP B-39/RTOG 0413, which compares $\mathrm{PBI}$ to whole breast irradiation, will provide cosmesis data in a more objective and large scale manner.

It should be noted that there is a consistently described relationship between cosmesis and balloon-skin surface distance. As this distance gets below the recommended $7 \mathrm{~mm}$ threshold, retraction and telangectasias become more prevalent, negatively affecting cosmesis.

\section{Tumor control}

The most basic endpoint of any radiation therapy treatment approach for breast cancer is its ipsilateral breast tumor control rate. The available data on this is as yet quite minimal due to the very recent development of the device. Published rates with very short follow-up are excellent, however. In the series with the largest number of patients [6] but with a median follow-up of only 5 months, 1 of 1237 patients $(<0.1 \%)$ developed an in-breast tumor recurrence - defined as a new primary breast cancer (presumably due its development in a different quadrant from the original cancer). Analysis of 158 patients treated at a variety of centers and reported by Choo et al. reveals a breast local recurrence rate of $0.6 \%$ at a median follow-up of 2 years [7]. The cohort with the longest follow-up is available from the patients treated prior to FDA approval in an effort to determine product safety and performance. This study, now with a median follow-up of 48 months, documented $100 \%$ tumor control within the breast in the 43 patients treated [8].

\section{Future directions}

The field of accelerated $\mathrm{PBI}$ is rapidly evolving, and PBI treatment approaches other than MammoSite are gaining acceptance (external beam 3D conformal radiotherapy, IMRT, and intra-operative radiotherapy). Since the local control, toxicity profile, and cosmesis scores associated with conventional whole breast radiotherapy are excellent, the bar is set quite high for the various $\mathrm{PBI}$ techniques to improve upon. Long term follow up of studies described above and the mature results of the currently accruing randomized trial (NSABP B-39/RTOG 0413) will help steer the course of $\mathrm{PBI}$ and determine the degree of future enthusiasm for this treatment approach.

\section{References}

1. Personal Communication from Dr. Frank Vicini for the American Society of Breast Surgeons, September 2006.

2. The American society of Breast Surgeons. Revised Consensus statement for accelerated partial breast irradiation. December 2005: http://www.breastsurgeons. org/apbi.shtml

3. NSABP. Protocol B-39; RTOG Protocol 0413: A randomized phase III study of conventional whole breast irradiation (WBI) versus partial breast irradiation (PBI) for women with stage 0 , I, or II breast cancer. http:// www.rtog.org/members/protocols/0413/0413.pdf

4. Suh WW, Pierce LJ, Vicini FA, et al. A cost comparison analysis of partial versus whole breast irradiation after breast-conserving surgery for early-stage breast cancer. Int J Radiat Oncol Biol Phys 2005; 62(3): 790-796.

5. Dowlatshahi K, Snider H, Gittleman M, et al. Early experience with balloon brachytherapy for breast cancer. Arch Surg 2004; 139: 603-608.

6. Vicini F, Beitsch P, Quiet C, et al. First analysis of patient demographics, technical reproducibility, cosmesis and early toxicity. Cancer 2005; 104(6): 1138-1148.

7. Choo J, Mehta P, Nguyen C, et al. Initial clinical results with the mammosite breast brachytherapy applicator in women with early-stage breast cancer [Abstract]. Int $J$ Radiat Oncol Phys 2005; 63(2) Supp1: 2002.

8. Keisch M, Vicini F, Scroggins T, et al. Thirty-nine month results with the mammosite brachytherapy applicator: details regarding cosmesis, toxicity and local control in partial breast irradiation [Abstract 10]. Radiat Oncol Phys 2005; 63(2) Supp1: (Data with 48 months median follow up presented at the proceedings of the American society for Therapeutic Radiology and Oncology, 47th Annual Meeting, October 2005). 